\title{
Factors responsible for the 2015 Cholera outbreak and spread in Ebonyi state, Nigeria
}

Onwe $\mathrm{FI}^{1}$, Agu $\mathrm{AP}^{2}$, Umezuruike $\mathrm{D}^{3}$ and Ogbonna $\mathrm{C}^{4}$

1. Department of Community Medicine, Federal Teaching Hospital, Abakaliki

2. Ebonyi State Ministry of Health, Abakaliki

3. Department of Community Medicine, Ebonyi State University

4. Department of Community Medicine, University of Jos

Correspondence: Onwe FI. email: fonwe012@gmail.com

\begin{abstract}
Background: Cholera outbreaks are frequent across Nigeria irrespective of season. Cholera outbreak began on the $17^{\text {th }}$ January 2015 in a community in Ebonyi State. We carried out an investigation to confirm the diagnosis, identify the source of infection, determine the factors associated with its spread and carried out some interventions.

Methods: Descriptive cross-sectional study was carried out in the affected communities during which active case search, environmental survey and key informant interviews were done. Stool samples were taken from cases for laboratory diagnosis. A cholera case was defined as any person that presented with diarrhoea and or vomiting with laboratory confirmation or with epidemiological linkage to the affected areas.

Results: A total of 551 cases were recorded in 38 communities with a case fatality rate of $8.6 \%$. Vibrio Cholera was isolated from 5 stool samples. Those who were 20 years or less and females were more commonly affected. Males and attendance at burial in another community within the two weeks preceding onset of outbreak was significantly associated with death $(p<0.001)$. Inadequate safe water supplies, poor sanitation and hygiene practices were observed.

Conclusion: The outbreak was caused by Vibrio Cholera. Socio-cultural practices surrounding burial such as overcrowding, ceremonial killing of animals and communal eating together may have contributed to its widespread. A system thinking approach such as behavior change communication and community dialogue are needed for Cholera control.
\end{abstract}

Key words: Cholera, Nigeria, socio-cultural, burial

Introduction

The tendency for Cholera caused by Vibrio Cholera to occur in explosive outbreaks and also cause pandemics are its two most distinguishing features. ${ }^{1}$ Cholera remains endemic (Fig. 1) in Africa, parts of Asia, the Middle East, South and Central America and is a key indicator of lack of social development which is closely correlated with high infant mortality. Oral and intravenous therapies are effective in acute diarrhoea caused by Cholera if instituted early. Cholera is a disease of major public health concern because it leads to death within hours if untreated. ${ }^{3}$ It is estimated that there are 1.44.3 million cases of Cholera resulting in 28,000-142,000 deaths worldwide yearly. ${ }^{4}$ In 2014 alone, the World Health Organization (WHO) reported a total of 190,549 cases of Cholera, $55 \%$ of which occurred in Africa with estimated 1.17\% fatality rate. ${ }^{5}$ 
In 2014, Nigeria recorded 35,996 suspected cases of Cholera with 306 laboratory confirmed cases and 755 deaths in 19 states and Federal Capital Territory (FTC) with Case Fatality Rate (CFR) of $2 \%{ }^{6}$ In 2015, 5,290 suspected cases were reported with 29 laboratory confirmed cases and 186 deaths involving 18 states and FCT giving a CFR of $3.5 \%{ }^{6}$ (Fig. 1 ). Factors which increased risk of Cholera transmission include inadequate environmental management with lack of access to safe water, poor sanitation, poor hygiene and over-crowding. Therefore, humanitarian crisis with disruption in water and sanitation systems, peri-urban slums, camps for Internally Displaced Persons (IDPs) that do not meet basic standards of clean water and sanitation, increase the risk of its spread. ${ }^{7}$

In 2010, Ebonyi State recorded 981 suspected cases of cholera with five laboratory confirmed cases and 22 deaths with CFR of $2.2 \%{ }^{8}$ In 2015 , there were 551 suspected cases with five laboratory confirmed cases and 64 deaths with CFR of $8.6 \%{ }^{8}$ (Fig.2). At the onset of the 2015 cholera outbreak in Ebonyi state, there was no published record of factors responsible for the spread. Understanding the factors responsible for its spread has become necessary to prevent future outbreaks through appropriate policy statements to guide the Public Health Sector. Therefore, the objective of this study was to identify the aetiological agent, source of infection and factors associated with its occurrence and spread.

\section{Methods}

Ebonyi state has 13 Local Government Areas (LGAs) and the inhabitants are mainly farmers, Civil servants, petty traders and artisans. The study was a descriptive cross-sectional design and interviewer administered questionnaire was used to obtain information from cases and household relatives of deceased cases. A total of 551 persons were interviewed out of which $53.7 \%$ were females. Data generated were on their sociodemography, symptoms, toilet facilities and burials attended within two weeks prior to an outbreak. Health facility-based surveillance from functional health centers reviewed medical records. Active case search using door-to-door surveillance was done in the communities affected. We determined the general sanitation of the communities affected, source of water supply and observed activities around the water bodies and personal hygiene practices through environmental surveys. Key Informant Interviews (KII) of thirty community opinion leaders and members were carried out. Informed consent was obtained from the key informants before the interview. Expedited prototype Ethical Clearance was obtained from the State Ministry of Health Ethical Committee. A case of cholera was defined as any person who presented with diarrhoea and or vomiting with laboratory confirmation or with epidemiological linkage to the affected areas (duration January to August 2015). Five stool samples from patients were tested with crystal VC immunechromatographic one step rapid visual test for Vibro Cholera (dipstick) on the field. Information from the outbreak investigation was shared with the communities and Key Informants. Statistical Package for Social Sciences (SPSS) version 20 was used to analyze the data obtained. Univariate analysis was done for frequency distribution, percentages, means, Standard deviation and CFRs statistical significance was set at $\mathrm{p} \leq 0.05$

\section{Results}

The age group under 10 years had the highest percentage of cases $(29.8 \%)$ 
followed by the age group 11-20 years $(19.4 \%)$. There was a statistically significant association between the outcome (alive or dead) and attendance to burials within the two weeks prior to onset of symptoms during the outbreak in the particular community $(\mathrm{p}<0.001)$.

There was also a statistically significant association between the outcome and gender. Being males was significantly associated with dying from the disease $(p=0.001)$. The other respondents' characteristics were not significantly associated with the outcomes. The age groups with the highest number of deaths were 11-20 years and 31-40 years. The number of cases was 551 out of which 64 deaths with case fatality rate of $8.6 \%$ occurred in the 38 communities of 10 LGAs of Ebonyi State.

Table 1: Characteristics of respondents and outcome (n-551)

\begin{tabular}{lcccc}
\hline \multicolumn{1}{c}{ Variable } & $\begin{array}{c}\text { Outcome } \\
\text { Alive } \\
\text { Freq. (\%) }\end{array}$ & $\begin{array}{c}\text { Dead } \\
\text { Freq. (\%) }\end{array}$ & $\chi^{2}$ & p-value \\
\hline $\begin{array}{l}\text { Attended burial } \\
\text { Yes }\end{array}$ & $17(21.5)$ & $62(78.5)$ & 401.6 & $<0.001$ \\
$\quad$ No & $470(99.6)$ & $2(0.4)$ & & \\
Gender & $213(83.5)$ & $42(16.5)$ & 10.9 & 0.001 \\
$\quad$ Male & $274(92.6)$ & $22(7.4)$ & & \\
Female & & & & \\
Type of toilet & $401(89.3)$ & $48(10.7)$ & & \\
Open & $86(84.3)$ & $16(15.7)$ & & \\
Pit & $278(86.6)$ & $43(13.4)$ & 2.374 & \\
Water source & $209(90.0)$ & $21(9.1)$ & & \\
Borehole & & & & \\
Stream & $38(97.4)$ & $1(2.6)$ & 11.586 \\
Age group (years) & $114(99.1)$ & $11(8.8)$ & & \\
$<1$ & $95(88.8)$ & $12(11.2)$ & & \\
$1-10$ & $72(85.7)$ & $12(14.3)$ & & \\
$11-20$ & $79(87.8)$ & $11(12.2)$ & & \\
$21-30$ & $53(89.8)$ & $6(10.2)$ & & \\
$31-40$ & $20(74.1)$ & $7(25.9)$ & & \\
$41-50$ & $16(80)$. & $4(20.0)$ & \\
$51-60$ & & & \\
$>60$ & &
\end{tabular}

Fig 1

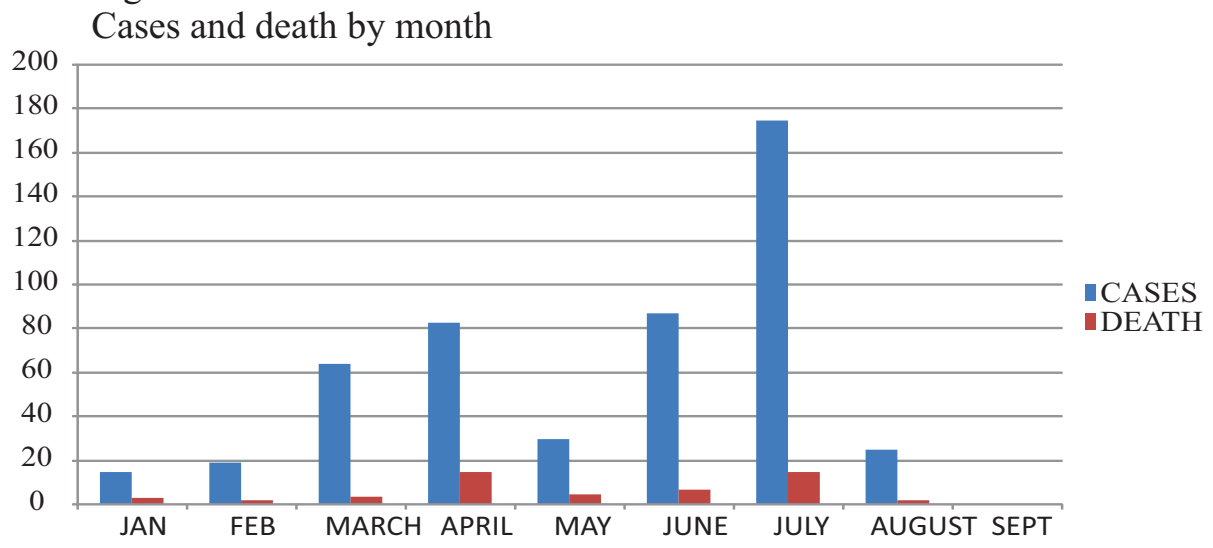




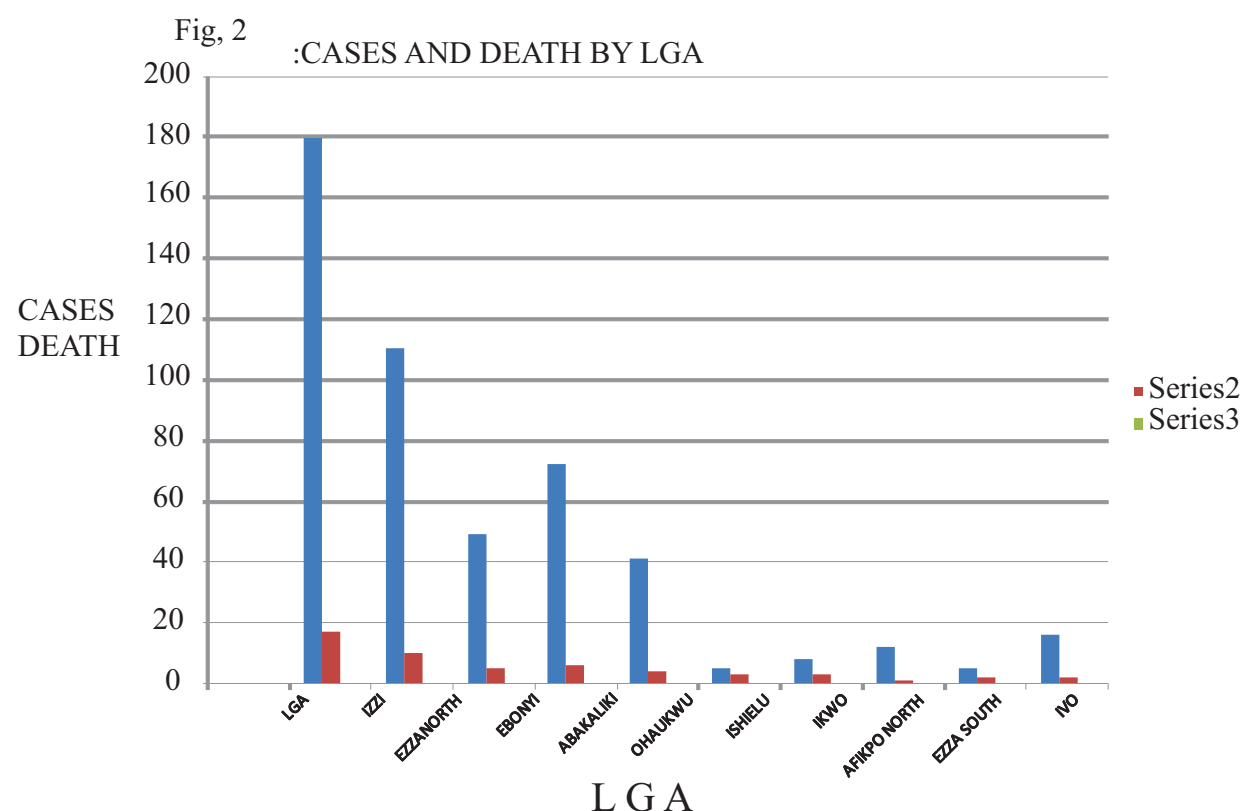

L G A

Discussion

The outbreak started on 15th January 2015 peaking in July and ended August $13^{\text {th }} 2015$ (4weeks after the last case was reported). In the remaining 37 affected communities (apart from that of the first index case), 29 of the index cases (each community had its own index case) had attended burial in another community within the two weeks prior to the onset of symptoms. The index case was in Ozibo community in Ebonyi LGA (a female market trader who had diarrhea and died two days later). Her source of drinking water was both stream and borehole. There was no history of any recent travels or attendance at burial. The outbreak of cholera spread around her community and to other communities followed the burial of this index case. The state ministry of health being notified on January 17th, 2015, the day of her death, immediately initiated the investigation of the outbreak.

Some of the Key Informants felt Cholera is caused by the gods and not bad water. That the dead must be buried in the usual socially accepted way with all the rites and people in attendance during outbreaks. A violation of this incurs the wrath of the gods on the family and community. Stream water tastes better than bore hole and the queues are discouraging.

The age groups most affected (under ten years and eleven to twenty years) in this study is similar to reports of children being mostly affected in northern and western parts of Nigeria. ${ }^{1,9,10}$ The greater proportion of deaths among the males may be because women generally utilize health facilities more than men and may readily seek care or likely be more knowledgeable on what to do at home.

The outbreak started in dry season and ended in rainy season. In Nigeria, outbreaks have been reported in both dry and rainy seasons. ${ }^{1}$ Attendance at a burial within the two weeks preceding the onset of symptoms was found to be significantly associated with the outcome of death among the cases. Twenty-nine of the thirty-eight index cases for the thirty eight communities, had attended burial in another community within the two weeks preceding the onset of symptoms and probably acquired the infection either from ingestion of contaminated food and water 
at the burial or practices like shaking of hands. The causes of death of the cases at the burials attended were all diarrheal diseases. At burials in Ebonyi state, like most parts of the country, the already existing conditions for transmission of water and food borne diseases is heightened due to overcrowding, slaughtering of animals for consumption, sharing and eating of food with poor hand washing practices, inadequate safe water supplies which increases the risk of Cholera spread.

The views of the opinion leaders interviewed were that the necessary rites and rituals must be carried out properly for burials. Health workers were not usually present to ensure hygienic practices such as disinfection of corpses before appropriate disposal, washing of hands with soap and water after touching the corpse, ensuring that people who handle the corpses are not involved in the preparation of food. Some of the sociocultural practices during burials may have been instrumental in the widespread of the 2015 cholera in Ebonyi State. Ensuring that food and water supply is not contaminated with human excreta is necessary for the prevention of Cholera. ${ }^{5}$ The movement of people from one community to another for burials, enhanced its spread. Population movement contributing to the spread of cholera has been documented in the report of a cholera outbreak in Niger state in 1995 where some residents who survived the initial outbreak (index case came from a neighbouring village) moved to other parts of the state and spread the infection there. ${ }^{11}$

The case fatality rate of $8.6 \%$ seen in this study is similar to that recorded in 2010 outbreaks in Kaduna 9\% and Kastina 7.6\% states which are higher than the 2015 outbreak average across the country that was $2.1 \%{ }^{1,6}$ High case fatality rates have been attributed to delay in seeking care, poor quality of care and wrong case definition. ${ }^{6}$ This could not be ascertained in this investigation.

Health education and radio programs during the outbreak advised against clustering of people at events but this was neither enforced nor adhered to by the people. Follow up community sensitization campaigns using trained members of the communities would encourage community partnership of health service delivery and may have yielded better results. Community measures to help control the spread of infection such as prompt disinfection of clothings and environment of patients could be better advocated for and followed up by trained members of the community .The use of trained community volunteers has been found useful in community based tuberculosis control activities. ${ }^{12}$ Inadequate safe water supplies, poor sanitation and unacceptable taste (salty) of borehole water seen in this study, were also documented as some of the socio-cultural risk factors for cholera in a study in Malawi. ${ }^{13}$

The actual exposed population could not be ascertained (those that attended the funeral of diarrhea disease victims). This was a limitation as only communities that had a Cholera outbreak were studied. There was no case control study. Sources of water supply and storage facilities of water in the households and community were neither tested for vibro cholera nor bacteriological analysis. The source of water used in cooking food was not established.

\section{Conclusion}

Nigeria is one of the countries in West Africa where Cholera is endemic (Fig. 1) 
despite repeated outbreaks and awareness about the disease. ${ }^{14}$ This study suggests that some of the socio-cultural practiced during burials were important factors in the spread of 2015 cholera outbreak in Ebonyi state. The study identified population movement during burial across communities of an index case, overcrowding, poor hygiene practices and inadequate water supply as some of the associated factors with the Cholera spread. This calls for community engagement towards sustained behavioural change, communication, adherence against mass gatherings during outbreaks, enhanced surveillance of diseases. Information from this study would be useful to Stakeholders in Public Health Sector for the prevention and control of Cholera outbreaks. It is a Systems thinking which demands a deeper understanding of the linkages, relationships, interactions and behaviour among the elements that characterize the entire system. ${ }^{1}$

\section{References}

1. Adagbada AO, Adesida SA, Nwaokorie FO et al. Cholera epidemiology in Nigeria: an overview. Pan African Medical Journal. 2012; 12:59.

2. Ackers M, Quick RE, Drasbek CJ et al. Are there national risk factors for epidemic cholera? The correlation between socioeconomic and demographic indices and cholera incidence in Latin America . International Journal of Epidemiology.1998; 27:330-334 3. Harris JB, Larocque RC, Qadri F, Calderwood SB. Cholera. Lancet. 2012; 379:2466-2476

4. Ali M, Lopez AL, AeYou Y, Eun Kim Y, Sah B, Maskerya B, Clemens J. The global burden of Cholera. Bull World Health Organ. 2012;90:209-218A 5. World Health Organization. Cholera 2014. Weekly epidemiological record. 2
Oct 2015; 90(40):517-544

6. Nigeria Centre for Disease Control (NCDC). Federal Ministry of Health. Weekly Epidemiological Report. 1st Jan. 2016, Vol. 5 No 52

7. World Health Organization. Cholera Fact sheet. No. 107. www.who.intaccessed 7th July 2016 8. Ebonyi State Ministry of Health Epidemiological unit. Report on Cholera. 2011

9. Hutin Y, Luby S, Paquet C. A large Cholera outbreak in Kano City, Nigeria: the importance of hand washing with soap and the danger of street vended water. Journal of Water and Health .2003; 01.1:45-52

10. Lawoyin TO, Ogunbodede NA, Olumide EAA, Onadeko MO. Outbreak of Cholera in Ibadan, Nigeria. European Journal of Epidemiology.1999;15:367370

11. Okeke IN, Abudu AB, Lamikara A. Microbiological investigation of an outbreak of acute gastroenteritis in Niger state, Nigeria. Clinical Microbiology and Infection. 2001;7 (9): 514-516

12. Balogun M, Sekoni A, Meloni ST. Odukoya O. Onajole A. Longe-Peters O. et al. Trained community volunteers improve Tuberculosis knowledge and attitudes among adults in a peri-urban community in Southwest Nigeria. Am. J. Trop. Med. Hyg. 2015; 92 (3): 625-632 13. Msyamboza KP, Kagoli M, M'bang'ombe M, Chipeta S Masuku H. Cholera outbreaks in Malawi in 19982012: social and cultural challenges in prevention and control. J Infect Dev Countries. 2014; 8(6): 720-726 14. Gaffga NH, Tauxe RV, Mintz ED. Cholera: A new homeland in Africa Am. J. Trop. Med. Hyg., 2007; 77(4): 705-713 15. De Savigny D, Adams T(eds). Systems Thinking for Health Systems Strengthening. Alliance for Health Policy and Systems Research, World Health Organization. Geneva. 2009 pp 33 